\title{
Short-term haptic memory for complex objects
}

\author{
MICHAEL J. KIPHART \\ St. Mary's College of Maryland, St. Mary's City, Maryland \\ and \\ JEFFREY L. HUGHES, J. PAUL SIMMONS, and HENRY A. CROSS \\ Mobile College, Mobile, Alabama
}

\begin{abstract}
College students actively felt complex objects for $3 \mathrm{sec}$, and, after delay intervals of $5,15,30$, or $45 \mathrm{sec}$, they received either the same object or a different object for comparison. In a signal detection framework, it was observed that $d^{\prime}$ scores were significantly higher for the 5-and 15 sec intervals than for the 30 - and 45 -sec intervals. Although a clearly negatively accelerated function was not obtained, this is one of the rare instances in which any decay function has been observed for haptic memory. Although it seems counterintuitive that the entire short-term-memory decay function occupies the narrow band extending from 15 to $30 \mathrm{sec}$, it is clear from previous studies that short-term haptic memory is unique. Results were discussed in terms of findings in both active- and passive-touch experiments.
\end{abstract}

After some inattention, the topic of tactual perception appears to be undergoing a revival of interest (Gordon, 1978; Schiff \& Foulke, 1982). Work has focused on both passive touch ("tactile" stimulation) and active touch (where "tactual" and "haptic" have been used synonymously).

In the active-touch domain, when short-term memory (STM) has been investigated, only Millar (1974), using children and complex stimuli, has reported an STM decay function as retention interval was systematically increased. Millar observed an increase in both errors and latencies with longer delays. Conversely, Klatzky, Lederman, and Metzger (1985) have argued that the identification of objects by touch involves an "expert" system, and Kiphart, Auday, and Cross (1988), in a series of studies, failed to find convincing evidence for an STM decay function even though they used a broad range of delay intervals and stimuli judged to be relatively complex. More recently, Bowers, Mollenhauer, and Luxford (1990) failed in their attempt to observe an STM decay function in a shared-attention recall task. However, in this latest study the range of intervals explored was somewhat narrow, that is, 5,10 , and $20 \mathrm{sec}$, and the shapes were simple and geometrical.

The present study was an effort to explore once again the possibility of an STM decay function in haptic memory while using a broader range of delay intervals and complex and homogeneous stimuli.

The authors wish to thank Andrew D. Kastello and Eun Young Lee for their ideas and assistance in constructing the stimulus objects. Henry A. Cross is Emeritus Professor of Psychology, Colorado State University, and Visiting Professor of Psychology, St. Mary's College of Maryland, for the academic year 1991-1992. Correspondence may be addressed to Michael J. Kiphart, Division of Human Development, St. Mary's College of Maryland, St. Mary's City, MD 20686.

\section{METHOD}

\section{Subjects}

Fourteen ( 9 female and 5 male) introductory psychology students from Mobile College volunteered to serve as subjects and were given extra credit for their participation. The total time involved for each was slightly more than $60 \mathrm{~min}$.

\section{Stimulus Materials}

The critical test stimuli were 32 complex three-dimensional configurations permanently affixed to a standard $7.6 \times 7.6 \times .6 \mathrm{~cm}$ Plexiglas base. The 32 basic patterns were developed using pieces taken from a LEGO building-block set. Each stimulus object was constructed using the same 15 standard pieces. The pieces were arranged into a completely filled $5.0-\mathrm{cm}^{2}$ first-level base pattern. A second level of pieces designed around the first-level base configuration allowed three individual LEGO pieces to be moved among the stationary pieces within individual four-position grids. The three individual pieces were single-element LEGO blocks that were randomly positioned within the respective fourposition grids to give each stimulus object a unique shape. Each object was identical except for the position of the three single-element individual pieces, which created unique objects within a homogeneous stimulus set.

\section{Apparatus}

The apparatus was constructed at Mobile College but was essentially the same as that employed in the Kiphart et al. study (1988). The apparatus allowed the subjects, who were all right-handed, to put their right hands through a sleeve-covered round aperture in a vertical opaque screen that separated the subject from the two contact experimenters. This apparatus differed from the prior one in that it had no track on which to slide the objects forward; rather, objects were always placed within a marked square to make it easy for the subjects to locate the objects, which they could not see.

\section{Procedure \\ The subjects were asked to actively touch a stimulus at the command "touch" and $3 \mathrm{sec}$ later to lift their fingers on the command "lift." After a delay interval, which was systematically manipulated, a second stimulus object was presented under exactly the same conditions, and after the "lift" command for that stimulus, the subjects were asked to respond either "same" or "different." The subjects were instructed to touch the tops of the objects with their fingertips and to respond "same" if the two successive stimuli were in fact identical but to respond "different" if the second stimulus had differed from the first one. A}


given stimulus was always presented in the same orientation. Care was taken to control carefully the touching time and to eliminate any differential auditory cues. One experimenter watched the timer and gave the auditory commands, while the second one selected the blocks as dictated by the data sheet and used the identical placement procedure for both same and different trials.

Each subject received four trials in a given block of trials, which consisted of two same and two different conditions that took place under the same delay interval. There were four delay intervals $(5,15,30$, and $45 \mathrm{sec}$ ) in all, and each basic condition was repeated for a total of six times. Accordingly, each subject received 96 total trials, or 24 blocks of four trials each. The order of blocks was randomized separately for each subject, with the one constraint being that sets of each time interval be completed as units. Within a given unit, the order of the two same and two different trials was randomized separately for each subject.

Each stimulus was numbered, and the order in which all 32 stimuli appeared was randomized separately for each subject. When a same condition was called for on a given trial, the experimenter simply presented the appropriate object successively, but when the different condition was in effect, the first and second stimuli were different and they were presented according to a predetermined random sequence that was different for each subject. All 32 objects were presented as the first object during the first 32 trials, and these same objects were repeated during trials 33-64 and 65-96. The second objects on the different trials were randomly selected without replacement. A given object always appeared in the same orientation with respect to the subject.

\section{RESULTS}

Responses were labeled "hits" (a response of " different" when two successive objects were in fact different) and "false alarms" (a response of "different" for the same condition); these were converted to $d$ ' scores, which constituted the basic datum in a randomized-block analysis of variance (ANOVA). In the overall analysis, the delay interval was significant $[F(3,39)=6.35, p<$ $.0013]$. The mean $d^{\prime}$ scores for the 5-, 15-, 30-, and 45sec conditions were, respectively, $1.45 \pm .90,1.64 \pm .75$, $.84 \pm .78$, and $.99 \pm .86$. Subsequent Duncan range comparisons $(\alpha=.01)$ showed that the 15 -sec condition produced significantly higher $d^{\prime}$ scores than did the 30- and 45-sec delay intervals. Furthermore, the 5-sec condition was significantly above the 30 -sec condition.

Another analysis with the same $d^{\prime}$ scores was done by comparing the 6 subjects having the highest average $d^{\prime}$ scores with the 6 having the lowest average $d^{\prime}$ scores in a split-plot ANOVA. Not surprisingly, the high and low groups differed $[F(1,10)=16.58, p<.002]$. Additionally, the delay intervals differed $[F(3,30)=6.05, p<$ $.0024]$, but there was no interaction between subject groups and delay intervals $[F(3,30)=.12, p<.95]$. Figure 1 depicts the findings involving the high and low subjects.

In the above $d^{\prime}$ score analysis, there were three scores that initially involved either a hit rate of 1.00 or a false alarm rate of .00 ; these were arbitrarily changed by .01 to allow the computation of $d^{\prime}$ scores. To allay any misgivings the reader might have about the changing of the values, all scores were subsequently converted to $A^{\prime}$ using the approach suggested by Johnson (1976). The overall $A^{\prime}$ analysis also showed a significant delay-interval effect $[F(3,39)=4.98, p<.005]$, and the $A^{\prime}$ means for the 5-, 15-, 30-, and 45-sec intervals were, respectively, $.80 \pm .10, .82 \pm .11, .70 \pm .17$, and $.70 \pm .16$. A post hoc

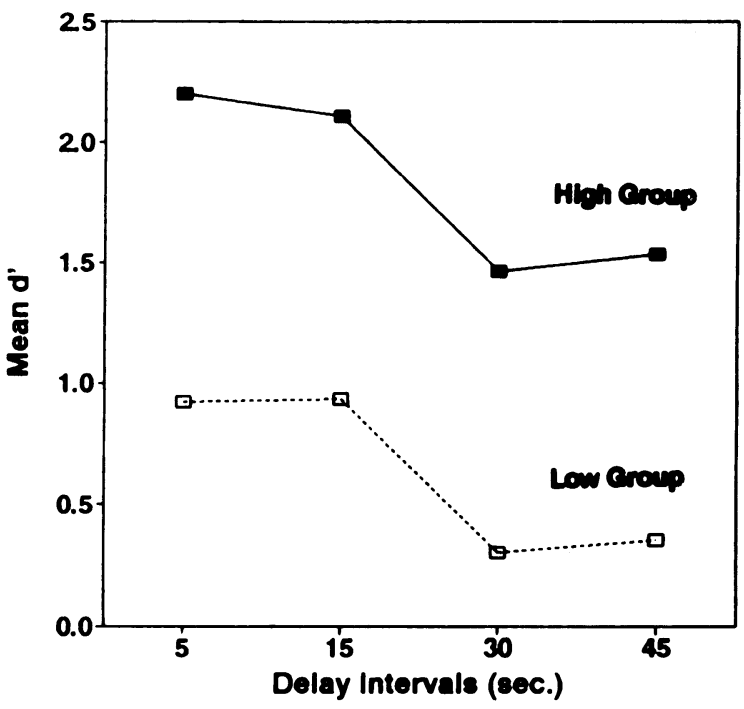

Figure 1. Mean d' scores for "high" and "low" subjects over the four delay intervals.

comparison revealed that the mean of the 15 -sec interval was significantly above that of the 30 - and $45-\mathrm{sec}$ intervals.

An analysis using $d^{\prime}$ measures of only the 6 highest subjects revealed a significant delay-interval effect $[F(3,15)$ $=34.02, p<.0001]$. The means for the 5-, 15-, 30-, and 45 -sec intervals were, respectively, $2.20 \pm .79$, $2.12 \pm .79,1.46 \pm .53$, and $1.53 \pm .75$. This particular analysis yielded results that most closely resembled a linear decay function across increasing delay intervals, although even here the mean for the 45 -sec interval was negligibly above that for the 30 -sec interval.

\section{DISCUSSION}

The lower overall mean $d^{\prime}$ scores in this experiment as compared with Kiphart et al. (1988) are putative evidence that this task, with its more complex objects drawn from a homogeneous object set and short touching times, provided a real test of a hypothesized haptic STM decay function. Even so, those subjects who were best, that is, the "high" group, performed respectably even at the longer intervals.

With respect to the matter of the STM decay function for the hapticsense modality, the issue remains unclear. Certainly there was a decline in $d^{\prime}$ and $A^{\prime}$ scores between the 15- and 30-sec intervals, and the fact that both "high" and "low" groups showed an almost identical function augurs well for the genuineness of this finding. Such a decline is unique in the experience of the first and last authors. On the other hand, the progressive decline with delay interval that one sees with the recall of other materials was simply not observed. Furthermore, it is counterintuitive to think that the entire decay function falls between 15 and $30 \mathrm{sec}$. However, in the matter of the haptic modality, we have come to expect the unexpected.

To summarize the data for active touch, it seems that only in this study with college students and complex objects and in a study by Millar (1974) with children and complex objects has anything resembling an STM decay function been observed. More often the result has been that of no decay function across various delay intervals and test procedures. In the passive-touch domain, Gilson and Baddeley (1969) stimulated the forearm and subsequently asked subjects to recall the stimulus location. They found that if subjects were required to count backwards by threes, their accuracy decreased up to $45 \mathrm{sec}$. However, Sullivan and Turvey (1972) 
replicated and extended the Gilson and Baddeley study and found no decay function, even to intervals of $60 \mathrm{sec}$.

If one is simply to compare those studies in which an STM decay function has been observed with those for which no such function has appeared, the box score clearly favors the latter group of findings. However, this does not seem like a satisfactory way of arriving at a conclusion.

\section{REFERENCES}

Bowers, R. L., Mollenhauer, M. S., \& LuXford, J. (1990). Shortterm memory for tactile and temporal stimuli in a shared-attention task. Perceptual \& Motor Skills, 70, 903-913.

Gilson, E. Q., \& BADDELEY, A. D. (1969). Tactile short-term memory. Quarterly Journal of Experimental Psychology, 21, 180-184.

Gordon, G. (ED.). (1978). Active touch. Oxford: Pergamon.

Johnson, N. S. (1976). A note on the use of $A^{\prime}$ as a measure of sensitivity. Journal of Experimental Child Psychology, 22, 530-531.
Kiphart, M. J., Auday, B. C., \& Cross, H. A. (1988). Short-term haptic memory for three-dimensional objects. Perceptual \& Motor Skills, 66, 79-91.

Klatzky, R. L., Lederman, S. J., \& Metzger, V. A. (1985). Identifying objects by touch: An "expert system." Perception \& Psychophysics, 37, 299-302.

MillaR, S. (1974). Tactile short-term memory by blind and sighted children. British Journal of Psychology, 65, 253-263.

SCHIFF, W., \& FoulKE, E. (1982). Tactual perception. Cambridge: Cambridge University Press.

Sullivan, E. V., \& TuRvey, M. T. (1972). Short-term retention of tactile stimulation. Quarterly Journal of Experimental Psychology, 24, 253-261.

(Manuscript received December 2, 1991.)

\section{Workshop \\ Memory Strategies: Theory and Application \\ University of Trieste \\ November 6-7, 1992}

A workshop on memory strategies will be held at the University of Trieste, November 6-7, 1992. The workshop is aimed at analyzing the role of cognitive strategies in storage and retrieval processes, and will focus on the main questions of the current debate: What is a "strategy"? What are the roles of attentional processes, metacognition factors, and intentions in adopting a memory strategy? What are the conditions that lead to the use of a specific strategy? What are the implications for a general model of the mind's architecture?

Invited speakers will include Graham Hitch, Asher Koriat, Michael Pressley, and Wolfgang Schneider. The workshop program will include oral presentations, guest speakers, and poster presentations.

The registration fee is 80,000 Italian Liras for participants and 40,000 Italian Liras for students. Payment of the fee should be addressed to PSY.CO 41554/0 Cassa di Risparmio di Trieste, Sede Centrale, Via Cassa di Risparmio 10, 34100 Trieste. 